\title{
Quantum Illumination with Simple Detection
}

\author{
Hao Yang*a, Wojciech Roga ${ }^{\mathrm{b}}$, Jonathan Pritchard ${ }^{\mathrm{a}}$, and John Jeffers ${ }^{\mathrm{a}}$ \\ ${ }^{a}$ CNQO, Dept. of Physics, University of Strathclyde, 107 Rottenrow, Glasgow G4 0NG, \\ United Kingdom
}

bNational Institute of Information and Communications Technology, 4-2-1 Nukui-Kitamachi, Tokyo 184-8795, Japan

\begin{abstract}
We show that by using the non-classical two-mode squeezed vacuum (TMSV) to illuminate an object, quantum correlations contribute to a detectable enhancement even under regimes of high signal loss and background thermal noise. We also consider a realistic measurement scenario with click detectors, along with sequential Bayesian inference; a single click on one mode of the TMSV produces a vacuum removed thermal state which enhances the probability of subsequent click detection.
\end{abstract}

\section{INTRODUCTION}

Entangled, or non-classically correlated states of the EM field are currently driving the frontiers of secure quantum key distribution ${ }^{1}$ and facilitate quantum teleportation ${ }^{2}$. In this report, we seek to extend applications of entangled states by using the strong correlations exhibited by the entangled two-mode squeezed vacuum for illumination purposes. The TMSV has a Fock basis expansion of

$$
|\Lambda\rangle=\sqrt{1-\lambda^{2}} \sum_{n} \lambda^{n}|n, n\rangle,
$$

where $\lambda=\tanh r$ and $r$ being the squeezing amplitude. Such entangled continuous variable (CV) field states may be created by non-degenerate parametric down-conversion in devices such as an $\mathrm{OPA}^{3}$, as $r$ is proportional to the nonlinear susceptibility of the down-converting crystal.

The class of quadrature squeezed states are a prime example of states where measurement precision beyond the standard quantum SNL is possible, without violating Heisenberg's uncertainty principle as the variance product is conserved $\sigma_{\hat{q}} \sigma_{\hat{p}}=\hbar^{2} / 4$. As for single mode squeezed states, variance along one quadrature may be decreased below the $\hbar / 2$ - at the expense of an increase along the perpendicular quadrature. But with the TMSV state, the variance is squeezed between two modes, along the axis of $\hat{q}_{1}-\hat{q}_{2}$ and $\hat{p}_{1}+\hat{p}_{2}$. In the limit of $r \rightarrow \infty$, one regains the EPR state ${ }^{4}$, which can violate Bell's inequality ${ }^{5}$. One can also use the photon number correlations of the TMSV to engineer Fock states or single photons.

Can we use quantum light to illuminate an object, and obtain a detectable advantage from the return signal - especially at low average photon numbers? Performing quantum illumination with entangled states requires

*e-mail: hao.yang@strath.ac.uk 
transmission of one mode whilst retaining the other. When the sent mode returns, some joint measurement is performed involving both modes. Due to the fragility of certain entangled states one may be correct to think advantages are unlikely, compared to classical illumination. However in the seminal paper ${ }^{6}$, Lloyd shows advantages for $d$-dimensional Bell states, before the theoretical treatment for continuous-variable Gaussian states ${ }^{7}$, suggesting that even under high losses with a weakly-reflective object, the TMSV outperforms the single mode coherent state (of similar average photon number, $\bar{n}$ ) under $M$ optimal discriminating detections. Such theoretical claims suggest quantum illumination would benefit stealthy surveillance, lidar or radar.

\section{CLICK DETECTION}

To produce the optimal discriminating measurement for two hypotheses $H_{0}$ (no object) and $H_{1}$ (object present), also known as the Helstrom measurement ${ }^{8}$, is difficult to implement physically. Conditional hypothesis states of the field may be characterised by density matrices $\rho_{0}$ and $\rho_{1}$, where the optimal discriminating POVM operator $\hat{\Pi}_{\text {opt }}$ must produce the sum of all positive eigenvalues of the matrix $\rho_{1}-\rho_{0}$ in order to minimise the average distinguishing (Bayesian) error, known as the Helstrom bound. The Helstrom bound is a theoretical limit, and experimental implementation would require bespoke measurement apparatus unsuitable for any other general measurements. It therefore makes more physical sense to use POVM operators which model physical detectors: we consider the on/off detector, producing results of click and no-click. The no-click POVM for a detector with quantum efficiency $\epsilon$ is written as

$$
\hat{\Pi}^{(0)}=\sum_{n}(1-\epsilon)^{n}|n\rangle\langle n|,
$$

which models commercially available avalanche photodiodes (APDs). The complementary click POVM is simply $\hat{\Pi}^{(1)}=\mathbb{1}-\hat{\Pi}^{(0)}$. Click detection only measures the diagonal elements of the density matrix, producing expectation values by $\operatorname{tr} \hat{\Pi} \rho$.

We test three CV Gaussian states of the same average photon number $\bar{n}$ against each other: coherent, TMSV and a two-mode thermal state. The reflection of the transmitted signal by an object with reflectivity $\eta$ is modelled by the beamsplitter transformation, which transforms the signal and idler annihilation operators by $\hat{a}_{s} \rightarrow \sqrt{\eta} \hat{a}_{s}+\sqrt{1-\eta} \hat{a}_{b}$ and $\hat{a}_{i}^{\prime} \rightarrow \hat{a}_{i}$, with $\hat{a}_{b}$ denoting the background noise the object is embedded in. The coincidence click probability is

$$
\left\langle\hat{\Pi}_{i}^{(1)} \hat{\Pi}_{s}^{(1)}\right\rangle_{\rho} \propto \int_{\mathbb{R}^{4}} d^{4} X W_{\hat{\Pi}_{i}^{(1)}} W_{\hat{\Pi}_{s}^{(1)}} W_{\rho},
$$

where a fully analytical solution can be obtained by computing the overlap integral using Wigner functions of the operators and states ${ }^{9}$ over their global phase-space (all states and operators have an equivalent Wigner representation $\left.{ }^{10}\right)$. Intuitively, if the object is present $\left(H_{1}\right)$ the global state $\rho_{1}$ has average photon number $\bar{n}_{i}+\eta \bar{n}_{s}+\bar{n}_{b}$ and if no object is present $\left(H_{0}\right), \rho_{0}$ would have average photon number $\bar{n}_{i}+\bar{n}_{b}$. But since strong non-classical correlations persist in the TMSV, compared to uncorrelated coherent states or classically correlated two-mode thermal states - the probability of coincidence will remain higher, even when $\left\langle\hat{n}^{\prime}\right\rangle_{\rho_{1}} \approx\left\langle\hat{n}^{\prime}\right\rangle_{\rho_{0}}$ where $\eta \ll 1$, as well as $\bar{n}_{b} \gg \bar{n}_{s, i}$. 


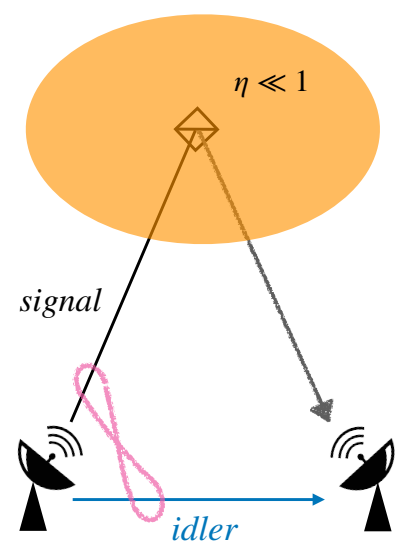

(a) Quantum Illumination

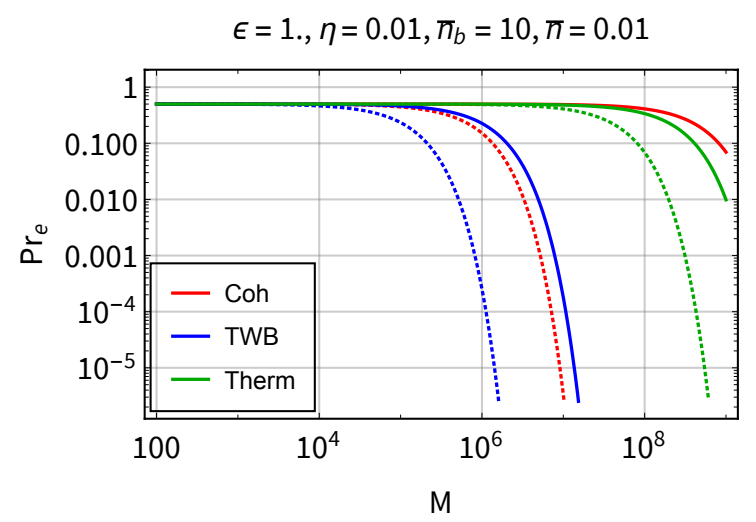

(b) Error Bounds

Figure 1: (a) - Diagram of quantum illumination: the signal and idler are entangled. (b) - Average Bayesian error bounds for coincidence detection as a function of repeated trials $M$. Dashed lines represent the optimal Helstrom measurement for each state, calculated using the quantum Chernoff bound $\frac{1}{2} \min _{0<s<1} \operatorname{tr}\left(\rho_{1}^{s} \rho_{0}^{1-s}\right)^{11}$. All three trial states have the same average photon number. The advantage of using TMSV for illumination is apparent as the error is decreasing at a higher rate, but click detection is suboptimal and the difference between the error exponents for TMSV curves is $10 \mathrm{~dB}$.

\section{SEQUENTIAL BAYESIAN RECEIVER}

We can estimate the probability of the object presence from click results by: $\tilde{p}_{1}=\operatorname{Pr}\left(\rho_{1} \mid \hat{\Pi}^{(i)}\right)$, then run multiple trials and calculate sequential posteriori probabilities by Bayes' rule

$$
\operatorname{Pr}(\rho \mid \hat{\Pi})=\frac{p \operatorname{tr} \hat{\Pi} \rho}{p \operatorname{tr} \hat{\Pi} \rho_{1}+(1-p) \operatorname{tr} \hat{\Pi} \rho_{0}},
$$

the probability $p$ is the a priori estimate of how probable there is a detectable object of interest (estimation of this with no measurement result is non-trivial). There are two possible measurement outcomes for both conditional states, with $\operatorname{Pr}_{D}=\operatorname{Pr}\left(\hat{\Pi}^{(1)} \mid \rho_{1}\right)=\operatorname{tr} \hat{\Pi}^{(1)} \rho_{1}$ the probability of successful object detection, and $\operatorname{Pr}_{F}=\operatorname{Pr}\left(\hat{\Pi}^{(1)} \mid \rho_{0}\right)=\operatorname{tr} \hat{\Pi}^{(1)} \rho_{0}$ as the probability of false-alarm. At each measurement trial, the detector will click with a probability $0<p_{c}<1$, which is deemed a click due to the object present by the following selection rule

$$
p_{c} \underset{\text { click }}{\stackrel{\text { no click }}{\gtrless}} \operatorname{Pr}_{D}
$$

meaning if $p_{c}<\operatorname{Pr}_{D}$, we calculate $\tilde{p}_{1}=\operatorname{Pr}\left(\rho_{1} \mid \hat{\Pi}^{(1)}\right)$, otherwise $\tilde{p}_{1}=\operatorname{Pr}\left(\rho_{1} \mid \hat{\Pi}^{(0)}\right)$. If no object is present, then we set $\operatorname{Pr}_{D}$ to $\operatorname{Pr}_{F}$ instead. Subsequent updates use the new prior $\tilde{p}_{1}$ to calculate the posterior, and so on.

If there are two modes - a heralding detection on the idler mode will occur with probability $\operatorname{tr}\left(\hat{\Pi}_{i} \otimes \mathbb{1}\right) \rho$, causing the partially-traced state $\mathcal{N} \operatorname{tr}_{i}\left(\hat{\Pi}_{i} \otimes \mathbb{1}\right) \rho$ to be transmitted. Such a state produced by the TMSV is a 
vacuum removed state, as the idler-click removes the vacuum

$$
\operatorname{tr}_{i}\left(\hat{\Pi}_{i} \otimes \mathbb{1}\right) \rho=\sum_{n} \lambda^{2 n}\left(1-\left(1-\epsilon_{i}\right)^{n}\right)|n\rangle\langle n|
$$

normalised by $\mathcal{N}=\left(1-\lambda^{2}\right)\left(1-\lambda^{2}\left(1-\epsilon_{i}\right)\right) / \epsilon_{i} \lambda^{2}$ - for $n=0$, the probability vanishes. This state increases $\operatorname{Pr}_{D}$ as average photon number in the signal arm is then raised by $\left(1-\lambda^{2}\left(1-\epsilon_{i}\right)\right)^{-1}$, and exactly one when the heralding efficiency is perfect.
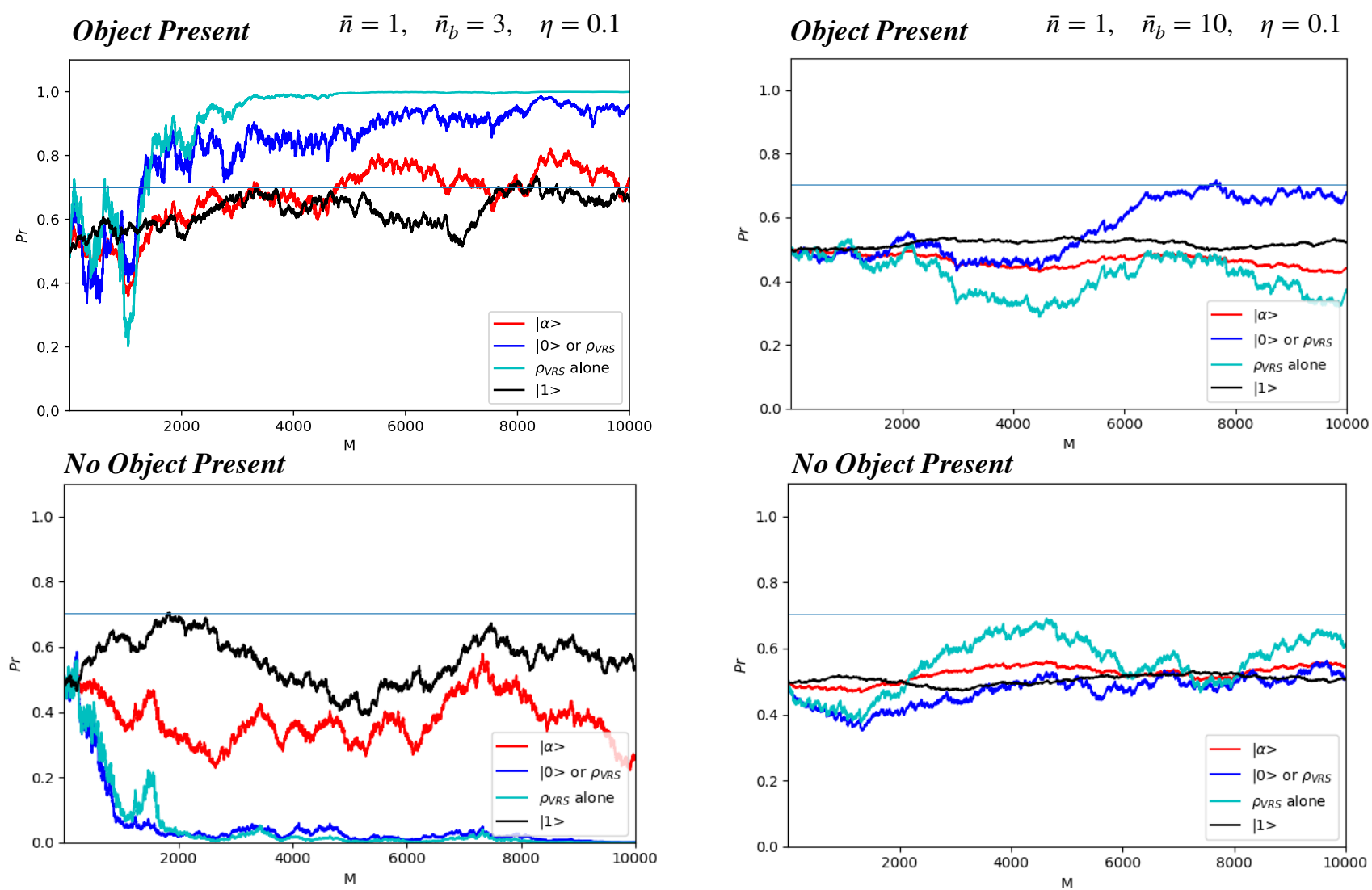

Figure 2: Monte-Carlo simulation of estimation curves for various signal test states via the sequential Bayesian receiver. The object is stationary, and the top row show trajectories with faster convergence for $\rho_{V R S}$ which is the conditioned vacuum removed state. Click detectors here have unit quantum efficiency. Traces follow estimates $\tilde{p}_{1}$ through each measurement trial $M$, where a new pseudorandom $\rho_{c}$ is generated. Thermal background is increasing with each column. $\rho_{V R S}$ is the vacuum removed state. " $|0\rangle$ or $\rho_{V R S}$ " would represent the TMSV with a heralding detector of perfect efficiency. Single photon states are also considered as a control. The constant line at $\operatorname{Pr}=0.7$ is an adjustable threshold, if one wish to label a detection before convergence. 


\section{DISCUSSION}

Our analysis here covers a basic framework for illumination using entangled quantum light, and demonstrates regimes where it outperfoms coherent states of the same energy. Despite the weakness of quantum signals, we can still show advantages provided by remaining correlations using simple click detection despite signal attenuation and background noise. In Section 3 we show that immediate detection of one arm of the TMSV, generating $\rho_{V R S}$ enhanced $\operatorname{Pr}_{D}$ shows faster convergence in the sequential receiver. The advantages may scale with typically preferential conditions such as low background noise, high signal photon number etc, however contrary to this we still see that TMSV outperforms the best possible classical states of the same energy. The main advantage of quantum illumination therefore, is stealth monitoring. The analysis provided here is perhaps more suited to lidar as non-classical light are routinely produced, but if applications were to extent to radar, then other experimental challenges would arise to create non-classical microwave states which involves superconducting elements. Our analysis relies purely on photon statistics alone and in theory it should be extendable to other frequencies.

\section{REFERENCES}

[1] Scarani, V., Bechmann-Pasquinucci, H., Cerf, N. J., Dušek, M., Lütkenhaus, N., and Peev, M., "The security of practical quantum key distribution," Rev. Mod. Phys. 81(3), 1301-1350 (2009).

[2] Bouwmeester, D., Pan, J. W., Mattle, K., Eibl, M., Weinfurter, H., and Zeilinger, A., "Experimental Quantum Teleportation," Nat. Phys. 390, 575-579 (1997).

[3] Ou, Z. Y., Pereira, S. F., Kimble, H. J., and Peng, K. C., "Realization of the Einstein-Podolsky-Rosen Paradox for Continuous Variables," Phys. Rev. Lett. 68(25), 3663-3666 (1992).

[4] Einstein, A., Podolsky, B., and Rosen, N., "Can Quantum-Mechanical Description of Physical Reality Be Considered Complete?," Phys. Rev. 47, 2-5 (1935).

[5] Banaszek, K. and Wódkiewicz, K., "Nonlocality of the Einstein-Podolsky-Rosen state in the Wigner representation," 58(6), 4345-4347 (1998).

[6] Lloyd, S., "Enhanced Sensitivity of Photodetection via Quantum Illumination," Science (80-. ). 321(5895), 1463-1465 (2008).

[7] Tan, S. H., Erkmen, B. I., Giovannetti, V., Guha, S., Lloyd, S., Maccone, L., Pirandola, S., and Shapiro, J. H., "Quantum illumination with gaussian states," Phys. Rev. Lett. 101(25), 1-4 (2008).

[8] Helstrom, C. W., "Quantum detection and estimation theory," J. Stat. Phys. 1(2), 231-252 (1969).

[9] Barnett, S. and Radmore, P., [Methods in Theoretical Quantum Optics], Oxford University Press (2003).

[10] Ferraro, A., Olivares, S., and Paris, M. G. A., [Gaussian states in continuous variable quantum information] (2005).

[11] Audenaert, K. M., Calsamiglia, J., Munoz-Tapia, R., Bagan, E., Masanes, L., Acin, A., and Verstraete, F., "Discriminating states: The quantum Chernoff bound," Phys. Rev. Lett. 98(16), 1-4 (2007). 